\title{
Closed-Loop Stabilization Over Gaussian Interference Channel ${ }^{\star}$
}

\author{
Ali A. Zaidi, Tobias J. Oechtering and Mikael Skoglund \\ School of Electrical Engineering and the ACCESS Linnaeus Center, \\ Royal Institute of Technology (KTH), Stockholm, Sweden. Emails: \\ $\{$ zaidi, oech, skoglund $\}$ @ee.kth.se.
}

\begin{abstract}
The problem of closed-loop stabilization of two scalar linear time invariant systems over symmetric white Gaussian interference channel with correlated noise components and arbitrarily distributed initial states is addressed. We propose to use linear and memoryless communication and control scheme based on the coding schemes introduced for interference networks which are an extension of the Schalkwijk-Kailath coding scheme. By employing the proposed communication and control scheme over the Gaussian interference channel, we derive sufficient conditions for mean square stability of the two linearly controlled LTI systems.
\end{abstract}

\section{INTRODUCTION}

The problem of remotely controlling dynamical systems over communication channels has gained significant attention in recent years. Such problems ask for interaction between stochastic control theory and information theory [Bansal and Basar (1989); Elia (2004)]. The minimum data rate below which the stability of an LTI system is impossible has been derived in stochastic and deterministic settings in [Nair and Evans (2002); Elia (2004); Yüksel (2010)], where they considered quantization errors and noise-free rate-limited channels. In [Tatikonda and Mitter (2004); Matveev and Savkin (2007)] are necessary rate conditions required to stabilize an LTI plant almost surely. However, from [Sahai and Mitter (2006)] we know that the characterization by Shannon capacity in general is not enough for sufficient conditions for moment stability in closed-loop control. In [Silva et al. (2008)], a simple coding scheme is proposed to mean square stabilize an LTI plant over noise-free rate-limited channels. The mean square stability of discrete plant over signal-to-noise ratio constraint channels is addressed in [Freudenberg et al. (2010)]. In [Yüksel and Basar (2011)], the authors considered noisy communication links between both observercontroller and controller-plant. In [Minero et al. (2009)], the necessary and sufficient conditions are derived for mean square stability of an LTI system over time varying feedback channels.

We formulate the general problem of controlling two scalar linear time invariant systems over the white Gaussian interference channel. The two user interference channel is a fundamental communication channel where two sources wish to communicate their messages to two different destinations and the signals transmitted from the sources interfere with each other [Carleial (1978)]. By the control over the interference we mean that there exist two separate sensors to sense the states of the two plants, and there exist two separate remote controllers to separately stabilize

\footnotetext{
$\star$ This work was supported in part by the European Commission through the FP7 project FeedNetBack (co-design for networked control systems).
}

the two plants i.e., a multi-sensor multi-controller setup. The capacity of the general interference channel is still an open problem, however the capacity region is known for some special cases. However in the context of closed-loop control, the interference channel with feedback is more relevant. In [Kramer (2002); Gastpar et al. (Submitted 2010)], the authors provided achievable rate regions over memoryless interference channel with noiseless and noisy feedback which is highly relevant to our problem. The coding schemes proposed by Kramer and Gastpar et al. in [Kramer (2002); Gastpar et al. (Submitted 2010)] for the memoryless Gaussian interference channels with noiseless feedback are adaptations of the well-known SchalkwijkKailath coding scheme for memoryless Gaussian pointto-point communication channel with noiseless feedback [Schalkwijk and Kailath (1966)].

We used the Schalkwijk-Kailath type scheme for deriving rate sufficient conditions for closed-loop stabilization of a scalar LTI sytem over white Gaussian relay channels in [Zaidi et al. (2010a, 2011)]. In [Zaidi et al. (2010b)], we used the Schalkwijk-Kailath based scheme to obtain stability regions for control over multiple-access and broadcast channels. In this paper we extend our previous work to the interference channel. We consider two scalar LTI plants with arbitrary distributed initial states which have to be remotely stabilized over a symmetric white Gaussian interference channel with correlated noise components. We use linear and memoryless communication and control policies based on Kramer's coding scheme to derive regions which are sufficient for mean square stability of the two plants in the absence of process and measurement noises.

\section{PROBLEM SETUP}

We consider two scalar discrete-time LTI systems whose state equations are given by

$$
X_{i, t+1}=\lambda_{i} X_{i, t}+U_{i, t}+W_{i, t}, \quad i=1,2,
$$

where $\left\{X_{i, t}\right\} \subseteq \mathbb{R},\left\{U_{i, t}\right\} \subseteq \mathbb{R}$, and $\left\{W_{i, t}\right\} \subseteq \mathbb{R}$, are state, control, and noise processes of the plant $i$. We assume that 


\section{MAIN RESULTS}

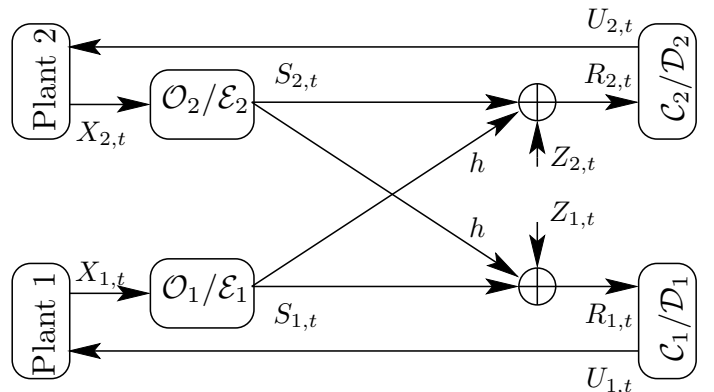

Fig. 1. The two unstable LTI plants have to be controlled over the white Gaussian interference channel with correlated noise components. There are two sensors to separately sense the states and two remotely located control units to separately control the two plants.

the initial states $X_{i, 0}$ are random variables with arbitrary probability distributions having variance $\alpha_{i, 0}=\mathbb{E}\left[X_{i, 0}^{2}\right]$ and correlation coefficient $\rho_{0}=\frac{\mathbb{E}\left[X_{1,0} X_{2,0}\right]}{\sqrt{\alpha_{1,0} \alpha_{2,0}}}$. The systems' parameters $\lambda_{i} \in \mathbb{R}_{+}$for all $i \in\{1,2\}$, and the $i$ th open loop system will be unstable if $\lambda_{i}>1$. We study the problem of remotely stabilizing the two systems over the white Gaussian interference channel.

The setup for control over symmetric white Gaussian interference channel is depicted in Fig. 1. There are two separate observers $\left\{\mathcal{O}_{1}, \mathcal{O}_{2}\right\}$ and separate controllers $\left\{\mathcal{C}_{1}, \mathcal{C}_{2}\right\}$ for the two plants. In order to communicate the observed state values to the controllers, an encoder $\mathcal{E}_{i}$ is lumped with the observer $\mathcal{O}_{i}$ and a decoder $\mathcal{D}_{i}$ is lumped with the controller $\mathcal{C}_{i}$. At any time instant $t$, the encoders $\mathcal{E}_{1}$ and $\mathcal{E}_{2}$ transmit $S_{1, t}$ and $S_{2, t}$ respectively. Accordingly the decoder $\mathcal{D}_{i}$ receives $R_{i, t}$ which is given by

$$
\begin{aligned}
& R_{1, t}=S_{1, t}+h S_{2, t}+Z_{1, t}, \\
& R_{2, t}=S_{2, t}+h S_{1, t}+Z_{2, t},
\end{aligned}
$$

where $h \in \mathbb{R}_{+}$is the cross channel gain, and $Z_{1, t} \sim$ $\mathcal{N}(0, N)$ and $Z_{2, t} \sim \mathcal{N}(0, N)$ are white noise components with a fixed cross-correlation coefficient $\rho_{z} \triangleq \frac{\mathbb{E}\left[Z_{1, t} Z_{2, t}\right]}{N}$ in the interval $[-1,1]$. The cross-correlation between the two noise components can model a common noise or common interference in the two signals. Let $f_{i, t}$ denote the $i$ th observer/encoder policy, then we have $S_{i, t}=$ $f_{i, t}\left(\left\{X_{i, k}\right\}_{k=0}^{t}\right)$ which must satisfy an average power constraint $\lim _{T \rightarrow \infty} \frac{1}{T} \sum_{t=0}^{T-1} \mathbb{E}\left[S_{i, t}^{2}\right] \leq P$. Further let $\gamma_{i, t}$ denote the $i$ th decoder/controller policy, then $U_{i, t}=$ $\gamma_{i, t}\left(\left\{R_{i, k}\right\}_{k=0}^{t}\right)$.

We assume that the process noise $W_{i, t}$ in (1) is zero, and focus on mean square stability [Nair and Evans (2002, 2004); Sahai and Mitter (2006); Freudenberg et al. (2006); Silva et al. (2008)] of the two plants. For a noise-free plant, we define mean square stability as follows.

Definition 1. A noise-free system is said to be mean square stable if and only if $\lim _{t \rightarrow \infty} \mathbb{E}\left[X_{t}^{2}\right]=0$, regardless of the initial state $X_{0}$.
We will first present our results in a comprehensive fashion and then provide the proofs in the next section.

Theorem 2. The two scalar LTI systems in (1) with $W_{i, t}=$ 0 can be mean square stabilized over the memoryless white Gaussian interference channel if the systems' parameters $\left\{\lambda_{1}, \lambda_{2}\right\}$ satisfy the following inequalities

$$
\log \left(\lambda_{i}\right)<\frac{1}{2} \log \left(\frac{P\left(1+h^{2}+2 h \rho^{\star}\right)+N}{P h^{2}\left(1-\rho^{\star 2}\right)+N}\right),
$$

where $\rho^{\star}$ is the largest among all the roots in the interval $[0,1]$ of the following two fourth order polynomials

$$
\begin{array}{r}
f_{1}(\rho):=\rho^{4}+a_{3} \rho^{2}+a_{2} \rho^{2}+a_{1} \rho+a_{0}, \\
f_{2}(\rho):=\rho^{4}+b_{3} \rho^{2}+b_{2} \rho^{2}+b_{1} \rho+b_{0},
\end{array}
$$

where

$$
\begin{aligned}
& a_{3}=\frac{N}{2 h P}, \quad a_{2}=-2-\frac{N\left(4+h \rho_{z}\right)}{2 h^{2} P}, \\
& a_{1}=-\frac{N\left(1+2 h^{2}+2 h \rho_{z}\right)}{2 h^{3} P}-\frac{N^{2}}{h^{3} P^{2}}, \\
& a_{0}=1+\frac{N\left(2 h-\rho_{z}\right)}{2 h^{3} P}, \quad b_{3}=\frac{2 h^{2} P+2 P+N}{2 h P}, \\
& b_{2}=\frac{N \rho_{z}}{2 h P}, \quad b_{1}=-\frac{\left(1+h^{2}\right)}{h}-\frac{N\left(1+2 \rho_{z}-2 h^{2}\right)}{2 h^{3} P}, \\
& b_{0}=-1-\frac{N\left(2 h-\rho_{z}\right)}{2 h^{3} P} .
\end{aligned}
$$

Proof. The proof is given in Sec. 4.

Remark 3. For fully correlated initial states, i.e., $\rho_{0}=1$, and fully correlated or anti-correlated noise components i.e., $\rho_{z}= \pm 1$, the initial transmissions in the proposed scheme in Sec. 4 can be modified such that $\rho^{\star}=1$. Accordingly the stability conditions are then given by

$$
\log \left(\lambda_{i}\right)<\frac{1}{2} \log \left(1+\frac{P(1+h)^{2}}{N}\right), \quad i=1,2 .
$$

Remark 4. It is shown in Appendix A that if the two noise components are fully correlated i.e., $\rho_{z}=1$, and further $2 h\left(1+\frac{h^{2} P}{N}\right)<1$, then the largest root $\rho^{\star}$ of the polynomial $f_{2}(\rho)$ is equal to one. Therefore the stability conditions are then given by

$$
\log \left(\lambda_{i}\right)<\frac{1}{2} \log \left(1+\frac{P(1+h)^{2}}{N}\right), \quad i=1,2 .
$$

Remark 5. The term on the right hand side in (2) corresponds to an achievable rate pair for the two sources over the white Gaussian interference channel with noiseless feedback [Gastpar et al. (Submitted 2010)].

\section{PROOF}

In order to prove Theorem 2 we propose a linear and memoryless communication and control scheme. This scheme is based on the well-known Schalkwijk-Kailath coding scheme [Schalkwijk and Kailath (1966); Kramer (2002); Gastpar et al. (Submitted 2010)]. By employing the proposed linear scheme over the given interference channel, we then find conditions on the system parameters $\left\{\lambda_{1}, \lambda_{2}\right\}$ which are sufficient to mean square stabilize the system in (1). The control and communication scheme for the interference channel works as follows. 
Initial time steps, $t=0,1$ Initially the two encoders transmit the observed state values in alternate time slots to the respective controllers. The first two disjoint transmissions in time make the plant states Gaussian distributed regardless of the distribution of their initial states, which will be explained shortly. However if the initial states are already Gaussian, then the following disjoint initial transmissions are not needed.

At time step $t=0$, the encoder $\mathcal{E}_{1}$ observes $X_{1,0}$ and transmits $S_{1,0}=\sqrt{\frac{P}{\alpha_{1,0}}} X_{1,0}$. The encoder $\mathcal{E}_{2}$ does not transmit, i.e., $S_{2,0}=0$. The decoder $\mathcal{D}_{1}$ receives $R_{1,0}=$ $S_{1,0}+Z_{1,0}$. It then estimates $X_{1,0}$ as

$$
\hat{X}_{1,0}=\sqrt{\frac{\alpha_{1,0}}{P}} R_{1,0}=X_{1,0}+\sqrt{\frac{\alpha_{1,0}}{P}} Z_{1,0} .
$$

The controller $\mathcal{C}_{1}$ then takes an action $U_{1,0}=-\lambda_{1} \hat{X}_{1,0}$ for the plant 1 , which results in $X_{1,1}=\lambda_{1}\left(X_{1,0}-\right.$ $\left.\hat{X}_{1,0}\right)$. The state $X_{1,1} \sim \mathcal{N}\left(0, \alpha_{1,1}\right)$ with $\alpha_{1,1}=\lambda_{1}^{2} \frac{\alpha_{1,0} N}{P}$. The controller does not take any action for the plant 2 , therefore $X_{2,1}=\lambda_{2} X_{2,0}$ with $\alpha_{2,1}=\lambda_{2}^{2} \alpha_{2,0}$.

At time step $t=1$, the encoder $\mathcal{E}_{1}$ does not transmit any signal. The encoder $\mathcal{E}_{2}$ observes $X_{2,1}$ and transmits $S_{2,1}=\sqrt{\frac{P}{\alpha_{2,1}}} X_{2,1}$. The decoder $\mathcal{D}_{2}$ receives $R_{2,1}=S_{2,1}+$ $Z_{2,1}$. It then estimates $X_{2,1}$ as

$$
\hat{X}_{2,1}=\sqrt{\frac{\alpha_{2,1}}{P}} R_{2,1}=X_{2,1}+\sqrt{\frac{\alpha_{2,1}}{P}} Z_{2,1} .
$$

The controller $\mathcal{C}_{2}$ then takes an action $U_{2,1}=-\lambda_{2} \hat{X}_{2,1}$ for the plant 2 , which results in $X_{2,2}=\lambda_{2}\left(X_{2,1}-\hat{X}_{2,1}\right)$. The state $X_{2,2} \sim \mathcal{N}\left(0, \alpha_{2,2}\right)$. For the plant 1 , the controller does not take any action $U_{1,1}=0$, therefore $X_{1,2}=\lambda_{1} X_{1,1}$ and $X_{1,2} \sim \mathcal{N}\left(0, \alpha_{1,2}\right)$.

It is noteworthy that due to non-overlapping initial transmissions by the two encoders, the states $X_{1,2}$ and $X_{2,2}$ are now zero mean Gaussian variables with correlation coefficient $\rho_{2}=\frac{\mathbb{E}\left[X_{1,2} X_{2,2}\right]}{\sqrt{\alpha_{1,2} \alpha_{2,2}}}$ equal to zero ${ }^{1}$. Henceforth the two encoders will transmit their signals simultaneously.

Further time steps $t \geq 2$ The two encoders $\mathcal{E}_{1}$ and $\mathcal{E}_{2}$ observe $X_{1, t}$ and $X_{2, t}$, and they respectively transmit

$$
S_{1, t}=\sqrt{\frac{P}{\alpha_{1, t}}} X_{1, t}, \quad S_{2, t}=\sqrt{\frac{P}{\alpha_{2, t}}} X_{2, t} \operatorname{sgn}\left(\rho_{t}\right),
$$

where $\rho_{t}=\frac{\mathbb{E}\left[\left(X_{1, t}-\mathbb{E}\left[X_{1, t}\right]\right)\left(X_{2, t}-\mathbb{E}\left[X_{2, t}\right]\right)\right]}{\sqrt{\alpha_{1, t} \alpha_{2, t}}}$ and $\operatorname{sgn}\left(\rho_{t}\right)=1$ if $\rho_{t} \geq 0$ and $\operatorname{sgn}\left(\rho_{t}\right)=-1$ if $\rho_{t}<0$.

In accordance, the decoder $\mathcal{D}_{1}$ receives $R_{1, t}=S_{1, t}+h S_{2, t}+$ $Z_{1, t}$ and the decoder $\mathcal{D}_{2}$ receives $R_{2, t}=S_{2, t}+h S_{1, t}+Z_{2, t}$. The decoder $\mathcal{D}_{i}$ then computes a memoryless ${ }^{2}$ MMSE estimate of the state of the plant $i$ as

\footnotetext{
1 The states in the second time step become uncorrelated irrespective of the value of the correlation between the initial states. This scheme does not exploit correlation between the initial states and thus the stability region obtained is independent of the correlation of the initial states.

2 The memoryless estimator is not optimal since the channel outputs are correlated. Therefore we expect that an improvement might be possible if we use full memory in the estimator. However the analysis becomes complicated by considering full LMMSE estimation.
}

$$
\hat{X}_{i, t}=\mathbb{E}\left[X_{i, t} \mid R_{i, t}\right] \stackrel{(a)}{=} \frac{\mathbb{E}\left[R_{i, t} X_{i, t}\right]}{\mathbb{E}\left[R_{i, t}^{2}\right]} R_{i, t},
$$

where $(a)$ follows from the fact that the optimum MMSE of the Gaussian variable is linear Hayes (1996); and we have

$$
\begin{aligned}
\mathbb{E}\left[X_{1, t} R_{1, t}\right] & =\sqrt{P \alpha_{1, t}}\left(1+h\left|\rho_{t}\right|\right), \\
\mathbb{E}\left[X_{2, t} R_{2, t}\right] & =\sqrt{P \alpha_{2, t}}\left(1+h\left|\rho_{t}\right|\right) \operatorname{sgn}\left(\rho_{t}\right), \\
\mathbb{E}\left[R_{i, t}^{2}\right] & =P\left(1+h^{2}+2 h\left|\rho_{t}\right|\right)+N .
\end{aligned}
$$

The controller $\mathcal{C}_{i}$ takes an action $U_{i, t}=-\lambda_{i} \hat{X}_{i, t}$ for the plant $i$, which results in $X_{i, t+1}=\lambda_{i}\left(X_{i, t}-\hat{X}_{i, t}\right)$. The mean values of the states are

$$
\begin{aligned}
\mathbb{E}\left[X_{i, t+1}\right] & =\mathbb{E}\left[\lambda_{i}\left(X_{i, t}-\hat{X}_{i, t}\right)\right] \\
& \stackrel{(a)}{=} \lambda_{i} \mathbb{E}\left[X_{i, t}-\frac{\mathbb{E}\left[R_{i, t} X_{i, t}\right]}{\mathbb{E}\left[R_{i, t}^{2}\right]} R_{i, t}\right] \stackrel{(b)}{=} 0,
\end{aligned}
$$

where $(a)$ follows from (4); and (b) follows from $\mathbb{E}\left[X_{i, 2}\right]=0$ and by recursively using $(a)$. The variance of the state $X_{i, t+1}$ is given by

$$
\alpha_{i, t+1} \triangleq \mathbb{E}\left[X_{i, t+1}^{2}\right]=\lambda_{i}^{2}\left(\mathbb{E}\left[X_{i, t}^{2}\right]-\frac{\left(\mathbb{E}\left[R_{i, t} X_{i, t}\right]\right)^{2}}{\mathbb{E}\left[R_{i, t}^{2}\right]}\right)
$$

By using (5) in (7) we get the following recursive equations

$$
\alpha_{i, t+1}=\alpha_{i, t} \lambda_{i}^{2}\left(\frac{P h^{2}\left(1-\left|\rho_{t}\right|^{2}\right)+N}{P\left(1+h^{2}+2 h\left|\rho_{t}\right|\right)+N}\right) .
$$

The cross-correlation coefficient $\rho_{t}$ between the two state processes for all $t \geq 3$ is given in (9) on the top of the next page. In the computation of $(9),(a)$ follows from

$$
\begin{aligned}
\mathbb{E}\left[X_{1, t} \hat{X}_{2, t}\right] & =\frac{\mathbb{E}\left[X_{1, t} R_{2, t}\right] \mathbb{E}\left[X_{2, t} R_{2, t}\right]}{\mathbb{E}\left[R_{2, t}^{2}\right]}, \\
\mathbb{E}\left[X_{2, t} \hat{X}_{1, t}\right] & =\frac{\mathbb{E}\left[X_{2, t} R_{1, t}\right] \mathbb{E}\left[X_{1, t} R_{1, t}\right]}{\mathbb{E}\left[R_{1, t}^{2}\right]}, \\
\mathbb{E}\left[\hat{X}_{1, t} \hat{X}_{2, t}\right] & =\frac{\mathbb{E}\left[X_{1, t} R_{1, t}\right] \mathbb{E}\left[X_{2, t} R_{2, t}\right] \mathbb{E}\left[R_{1, t} R_{2, t}\right]}{\mathbb{E}\left[R_{1, t}^{2}\right] \mathbb{E}\left[R_{2, t}^{2}\right]},
\end{aligned}
$$

(b) follows from

$$
\begin{aligned}
& \mathbb{E}\left[X_{1, t} R_{2, t}\right]=\sqrt{P \alpha_{1, t}}\left(h+\left|\rho_{t}\right|\right), \\
& \mathbb{E}\left[X_{2, t} R_{1, t}\right]=\sqrt{P \alpha_{2, t}}\left(h+\left|\rho_{t}\right|\right) \operatorname{sgn}\left(\rho_{t}\right), \\
& \mathbb{E}\left[R_{1, t} R_{2, t}\right]=2 h P+P\left|\rho_{t}\right|\left(1+h^{2}\right)+N \rho_{z},
\end{aligned}
$$

(c) follows from (8); and $(d)$ follows by defining $g\left(\rho_{t}\right)$

Now we wish to find conditions on the parameters $\left\{\lambda_{1}, \lambda_{2}\right\}$ which ensure mean square stability of the two systems in (1) over the given white Gaussian interference channel. In order to find the values of the parameters $\left\{\lambda_{1}, \lambda_{2}\right\}$ for which the variance of the two state processes given by (8) can be made equal to zero as time goes to infinity, we make use of the following lemma.

Lemma 6. For the recursive equation in (9) there exists at least one $\rho^{\star} \in[0,1]$ such that if $\left|\rho_{t}\right|=\rho^{\star}$ then $\left|\rho_{t+k}\right|=\rho^{\star}$ for all $k \geq 0$, where $\rho^{\star}$ is a root of one of the two polynomials $\left\{f_{1}(\rho), f_{2}(\rho)\right\}$ given in (3). Further if $\rho^{\star}$ is a root of $f_{1}(\rho)$ then $\rho_{t+k}=(-1)^{k} \rho^{\star}$, and if $\rho^{\star}$ is a root of $f_{2}(\rho)$ then $\rho_{t+k}=\rho^{\star}$ for all $k \geq 0$.

Proof. The proof can be found in Appendix A. 


$$
\begin{aligned}
& \rho_{t+1}= \frac{\mathbb{E}\left[X_{1, t+1} X_{2, t+1}\right]}{\sqrt{\alpha_{1, t+1} \alpha_{2, t+1}}}=\frac{1}{\sqrt{\alpha_{1, t+1} \alpha_{2, t+1}}} \mathbb{E}\left[\lambda_{1}\left(X_{1, t}-\hat{X}_{1, t}\right) \lambda_{2}\left(X_{2, t}-\hat{X}_{2, t}\right)\right] \stackrel{(a)}{=} \frac{\lambda_{1} \lambda_{2}}{\sqrt{\alpha_{1, t+1} \alpha_{2, t+1}}} \\
& \times\left(\mathbb{E}\left[X_{1, t} X_{2, t}\right]-\frac{\mathbb{E}\left[X_{1, t} R_{2, t}\right] \mathbb{E}\left[X_{2, t} R_{2, t}\right]}{\mathbb{E}\left[R_{2, t}^{2}\right]}-\frac{\mathbb{E}\left[X_{2, t} R_{1, t}\right] \mathbb{E}\left[X_{1, t} R_{1, t}\right]}{\mathbb{E}\left[R_{1, t}^{2}\right]}+\frac{\mathbb{E}\left[X_{1, t} R_{1, t}\right] \mathbb{E}\left[X_{2, t} R_{2, t}\right] \mathbb{E}\left[R_{1, t} R_{2, t}\right]}{\mathbb{E}\left[R_{1, t}\right] \mathbb{E}\left[R_{2, t}\right]}\right) \\
& \stackrel{(b)}{=} \lambda_{1} \lambda_{2} \sqrt{\frac{\alpha_{1, t} \alpha_{2, t}}{\alpha_{1, t+1} \alpha_{2, t+1}}}\left(\rho_{t}-2 \frac{P \operatorname{sgn}\left(\rho_{t}\right)\left(h+\left|\rho_{t}\right|\right)\left(1+\left|\rho_{t}\right|\right)}{P\left(1+h^{2}+2 h\left|\rho_{t}\right|\right)+N}+\frac{P \operatorname{sgn}\left(\rho_{t}\right)\left(1+h\left|\rho_{t}\right|\right)^{2}\left(2 h P+P\left|\rho_{t}\right|\left(1+h^{2}\right)+N \rho_{z}\right)}{\left(P\left(1+h^{2}+2 h\left|\rho_{t}\right|\right)+N\right)^{2}}\right) \stackrel{(c)}{=} \\
& \operatorname{sgn}\left(\rho_{t}\right) \underbrace{\left(\frac{P\left(1+h^{2}+2 h\left|\rho_{t}\right|\right)+N}{P h^{2}\left(1-\left|\rho_{t}\right|^{2}\right)+N}\right)\left(\left|\rho_{t}\right|-2 \frac{P\left(h+\left|\rho_{t}\right|\right)\left(1+\left|\rho_{t}\right|\right)}{P\left(1+h^{2}+2 h\left|\rho_{t}\right|\right)+N}+\frac{P\left(1+h\left|\rho_{t}\right|\right)^{2}\left(2 h P+P\left|\rho_{t}\right|\left(1+h^{2}\right)+N \rho_{z}\right)}{\left(P\left(1+h^{2}+2 h\left|\rho_{t}\right|\right)+N\right)^{2}}\right)}_{\triangleq g\left(\rho_{t}\right)}
\end{aligned}
$$

$\stackrel{(d)}{=} \operatorname{sgn}\left(\rho_{t}\right) \cdot g\left(\rho_{t}\right), \quad \forall t \geq 2$

If we modify our encoding scheme such that $\rho_{2}$ becomes equal to $\rho^{\star}$ instead of zero, then $\left|\rho_{t}\right|$ will be equal to $\rho^{\star}$ for all $t \geq 2$. This modification ${ }^{3}$ in the encoding scheme can be done as follows. Suppose in the initial transmissions (i.e., $t=0,1$ ) the two encoders transmit $S_{1,0}=\sqrt{\frac{P}{\alpha_{1,0}}} X_{1,0}+m$ and $S_{2,1}=\sqrt{\frac{P}{\alpha_{2,1}}} X_{2,1}+m$, where $m$ is a Gaussian variable with zero mean and variance $\sigma_{m}^{2}$. In this way $\rho_{2}$ can take on any value between zero and one by varying $\sigma_{m}^{2}$. Thus by choosing $\sigma_{m}^{2}$ such that $\rho_{2}=\rho^{\star}$, we can rewrite (8) as

$$
\begin{aligned}
\alpha_{i, t+1} & =\alpha_{i, t} \lambda_{i}^{2}\left(\frac{P h^{2}\left(1-\rho_{t}^{\star 2}\right)+N}{P\left(1+h^{2}+2 h \rho_{t}^{\star}\right)+N}\right) \\
& =\alpha_{i, 2}\left(\lambda_{i}^{2} \frac{P h^{2}\left(1-\rho_{t}^{\star 2}\right)+N}{P\left(1+h^{2}+2 h \rho_{t}^{\star}\right)+N}\right)^{t-2} .
\end{aligned}
$$

Although in the modified encoding scheme we have violated the average power constraint for the first two transmissions, its effect can be neglected for infinite time horizon. We observe from (11) that $\alpha_{i, t} \rightarrow 0$ as $t \rightarrow \infty$ if

$$
\begin{aligned}
& \left(\lambda_{i}^{2} \frac{P h^{2}\left(1-\rho_{t}^{\star 2}\right)+N}{P\left(1+h^{2}+2 h \rho_{t}^{\star}\right)+N}\right)<1 \\
& \Rightarrow \log \left(\lambda_{i}\right)<\frac{1}{2} \log \left(\frac{P\left(1+h^{2}+2 h \rho^{\star}\right)+N}{P h^{2}\left(1-\rho^{\star 2}\right)+N}\right),
\end{aligned}
$$

for $i$ in $\{1,2\}$. The term on the right hand side in (12) is a monotonically increasing function of $\rho^{\star}$, therefore we choose $\rho^{\star}$ to be the largest among all roots in $[0,1]$ of the two polynomials $\left\{f_{1}(\rho), f_{2}(\rho)\right\}$. The condition on $\lambda_{i}$ in (12) guarantees mean square stability of the $i$ th the open loop system if it is unstable i.e., $\lambda_{i}>1$. For $\lambda_{i}<1$ (i.e., $\left.\log \left(\lambda_{i}\right)<0\right)$, the open loop system is self stable and the variance of the state process will converge to zero without any control actions in closed-loop. Therefore the sufficient conditions for mean square stability are given by (2). This completes the proof of Theorem 2 .

\footnotetext{
3 At this point we modify the encoding scheme in order to artificially guarantee convergence of $\rho_{t}$ to a fixed point. Numerical experiments suggest that $\rho_{t}$ always converges to a fixed point starting from an arbitrary $\rho_{2}$, and this fixed point is unique.
}

\section{NUMERICAL RESULTS AND DISCUSSION}

According to Theorem 2, the stabilizability of the two first order LTI systems depends on the given interference channel parameters such as average transmit power $P$, noise power $N$, noise cross-correlation $\rho_{z}$, and cross channel gain $h$. Therefore it is interesting to study the effect of these channel parameters on the behavior of the two systems under our proposed communication and control scheme. In this section we investigate the stabilizability of the two systems under our proposed scheme for different values of noise cross-correlation and cross channel gain with fixed transmit and noise powers.

In Fig. 2 we fix $P=20, N=1$, and plot the boundary of the stability region for the two plants as a function of $\rho_{z}$ for different values of $h$, according to Theorem 2 . Therefore the $i$ th plant will be mean square stable under our proposed scheme for the given channel parameters if $\log \left(\lambda_{i}\right)$ is in the region below the corresponding stability boundary curve. In Fig. 2 we have shown some examples for different levels of interference parameter, i.e., $h=$ $\{0,0.15,1,100\}$. In most cases stability region reduces by increasing $\rho_{z}$ from -1 to 1 , except for the case when the interference is very weak, i.e. $h=0.15$. For this case, it is given in Remark 4 that the best performance is achieved when $\rho_{z}=1$. For the sake of comparison we have also plotted no interference case $(h=0)$, i.e., there exist two parallel channels from the two plants to the two remote controllers. For this setup the stability conditions are given by $\log \left(\lambda_{i}\right)<0.5 \log (1+P / N)$. In Fig. 2 we have also shown an example of very strong interference scenario $(h=100)$. This example suggests that the stability region significantly expands in the presence of a very strong interference. In order to investigate this further, we now fix $P=20, N=1$, and plot the boundary of the stability region as a function of $h$ for different values of $\rho_{z}$ in Fig. 3 . For $\rho_{z}=\{-1,-0.95\}$ the stability increases monotonically with increasing cross channel gain $h$. Interestingly we observe a boost in the stability of the systems for $\rho_{z}=-1$ compared to $\rho_{z}=-0.95$ in the high interference regime. For $\rho_{z}=\{0,1\}$ the worst performance happens when the interference is moderate (i.e., neither weak nor strong). However in these examples too the stability improves monotonically with increase in cross channel gain beyond certain threshold. Further we observe that in the low 
interference regime (i.e. for very small values of $h$ ) the best performance is achieved when the noise components are fully correlated $\left(\rho_{z}=1\right)$, which is in accordance with Remark 4.

In the given setup of control over interference channel, the two systems are driven by the actions of the two controllers. These control actions are influenced by the cross channel interference, therefore it is also interesting to study cross-correlation between the state processes of the two systems for different values of cross channel gain. Under our proposed scheme, the magnitude of the crosscorrelation coefficient between the two state processes remains constant in the steady state, however it might alternate in phase in successive time steps as shown in Sec. 4. In Fig. 4 we fix $P=20, N=1$, and plot magnitude of the state cross-correlation coefficient $\rho^{\star}$ as a function of cross channel gain $h$ for different values of noise correlation $\rho_{z}=\{-1,-0.5,0,1\}$. In these examples, we observe a general trend that cross correlation increases in magnitude as cross channel gain increases. The state processes of the two systems become almost fully correlated as cross channel interference gets very strong. This happens because the two systems are driven control actions which are highly influenced by cross talk.

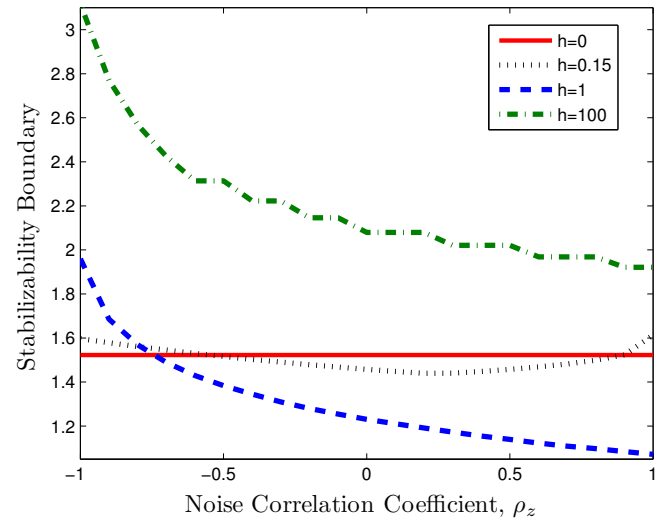

Fig. 2. Examples of the stabilizability region of the two systems as a function of cross-correlation coefficient of the two white noise components in the symmetric Gaussian interference channel, with fixed $P=20$, $N=1$.

In summary, the above numerical examples suggest that the stability region of the two systems over Gaussian interference channel reduces with the increase of noise cross-correlation from -1 to +1 . That is negative correlation helps and positive correlation hurts except for the case when interference is very weak (see also Remark $4)$. Further for anti-correlated $\left(\rho_{z}=-1\right)$ noises there is a dramatic boost in the stabilizability especially in the presence of strong interference. A similar behavior was observed in [Gastpar et al. (Submitted 2010)], where the authors showed that the sum-rate capacity over symmetric Gaussian interference channel can be doubled with feedback in high SNR when the noise components are anticorrelated. Furthermore we have observed that in general stabilizability under the proposed scheme improves as the interference gets significantly stronger. This result is in line with already known results in information theory, where it has been shown that the transmission rates over inter-

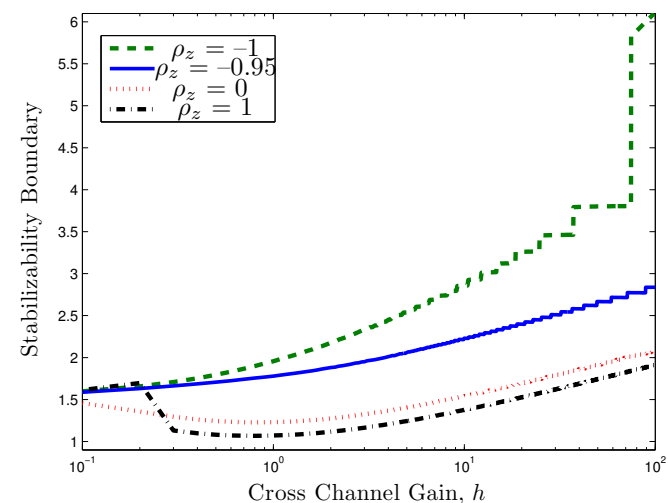

Fig. 3. Examples of the stabilizability region of the two systems as a function of cross channel gain in the symmetric Gaussian interference channel, with fixed $P=20, N=1$.

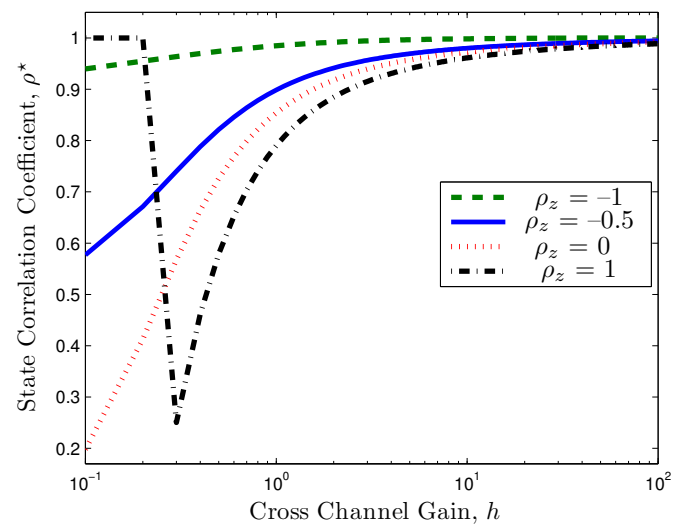

Fig. 4. Illustration of cross correlation coefficient of the state processes of the two systems in steady state as a function of cross channel gain for different values of noise correlation, with fixed $P=20, N=1$.

ference channel can be significantly improved in presence of very strong interference [Sato (1981)]. Further we have observed that the state processes of the two systems become highly correlated in magnitude in strong interference scenarios under our proposed scheme.

The stability results provided in this paper can be extended for non-symmetric interference channel using the proposed scheme with some tedious computations. We can also extend our results for the setup where the links from the controllers to the plants are also white Gaussian communication channels. For this setup we can have an encoder at each control unit to encode the control action and a MMSE decoder at each plant to decode the transmitted value of the control action. As long as the encoders, the decoders, and the controllers are linear, the nature of the problem does not change and the stability results can be easily obtained cf. [Yüksel and Basar (2011)].

\section{REFERENCES}

Bansal, R. and Basar, T. (1989). Simultaneous design of measurement and control strategies for stochastic systems with feedback. Automatica, 25(5), 679-694.

Carleial, A. (1978). Interference Channels. IEEE Trans. Inform. Theory, 24(1), 60-70. 
Elia, N. (2004). When Bode meets Shannon: controloriented feedback communication schemes. IEEE Trans. on Automat. Control, 49(9), 1477-1488.

Freudenberg, J., Middleton, R., and Solo, V. (2006). The minimal signal-to-noise ratio rqeuired to stabilize over a noisy channel. In Proc. $A C C$.

Freudenberg, J., Middleton, R., and Solo, V. (2010). Stabilization and disturbance attenuation over a Gaussian communication channel. IEEE Trans. Automat. Control, 55(3), 795-799.

Gastpar, M., Lapidoth, A., and Wigger, M. (Submitted 2010). When feedback doubles the prelog in AWGN networks. IEEE Trans. Inform. Theory. URL http://arxiv.org/abs/1003.6082.

Hayes, M. (1996). Statistical digital signal processing and modelling. John Wiley Sons, Inc.

Kramer, G. (2002). Feedback strategies for white gaussian interference networks. IEEE Trans. Inform. Theory, 48(6), 1423-1438.

Matveev, A. and Savkin, A. (2007). An analogue of shannon information theory for detection and stabilization via noisy discrete communication channels. SIAM J. Control Optim., 46(4), 1323-1367.

Minero, P., Franceschetti, M., Dey, S., and Nair, G.N. (2009). Data rate theorem for stabilization over time varying feedback channels. IEEE Trans. Automat. Control, 54(2), 243-255.

Nair, G.N. and Evans, R.J. (2002). Mean square stabilisability of stochastic linear systems with data rate constraints. In Proc. IEEE CDC, 1632-1637.

Nair, G.N. and Evans, R.J. (2004). Stabilizability of stochastic linear systems with finite feedback data rates. SIAM J. Control Optim., 43(2), 413-436.

Sahai, A. and Mitter, S. (2006). The necessity and sufficiency of anytime capacity for stabilization of a linear system over noisy communication links-part i: Scalar systems. IEEE Trans. Inform. Theory, 52(8), 3369-3395.

Sato, H. (1981). The capacity of the Gaussian interference channel under strong interference. IEEE Trans. Inform. Theory, 27(6), 786 - 788. doi: 10.1109/TIT.1981.1056416.

Schalkwijk and Kailath, T. (1966). A coding scheme for additive noise channels with feedback-I: No bandwidth constraint. IEEE Trans. Inform. Theory, 12(2), 172182.

Silva, E., Derpich, M., Ostergaard, J., and Quevedo, D. (2008). Simple coding for achieving mean square stability over bit-rate limited channels. In Proc. IEEE CDC, 2698-2703.

Tatikonda, S. and Mitter, S. (2004). Control over noisy channels. IEEE Trans. Automat. Control, 49(7), 11961201.

Yüksel, S. (2010). Stochastic stabilization of noisy linear systems with fixed-rate limited feedback. IEEE Trans. Automat. Control, $\mathrm{PP}(99)$.

Yüksel, S. and Basar, T. (2011). Control over noisy forward and reverse channels. IEEE Trans. Automat. Control, 56.

Zaidi, A.A., Oechtering, T.J., and Skoglund, M. (2010a). Rate sufficient conditions for closed-loop control over AWGN relay channels. In IEEE ICCA, 602-607.
Zaidi, A.A., Oechtering, T.J., and Skoglund, M. (2010b). Sufficient conditions for closed-loop control over multiple-access and broadcast channels. In IEEE CDC, 4771-4776.

Zaidi, A.A., Oechtering, T.J., Yüksel, S., and Skoglund, M. (2011). Sufficient conditions for closed-loop control over a Gaussian relay channel. In IEEE $A C C$.

\section{Appendix A. PROOF OF LEMMA 6}

Consider the recursive equation $\rho_{t+1}=\operatorname{sgn}\left(\rho_{t}\right) g\left(\rho_{t}\right)$ given in $(9)$. Based on $g(\rho)$ we define two polynomials $\left\{f_{1}(\rho), f_{2}(\rho)\right\}$ as,

$$
f_{1}(\rho):=l_{1}(\rho+g(\rho)), \quad f_{2}(\rho):=l_{2}(-\rho+g(\rho)),
$$

where $l_{1}$ and $l_{2}$ are two non-zero scalars chosen such that the leading coefficients of the two polynomials are equal to one, i.e., $f_{1}(\rho)$ and $f_{2}(\rho)$ are monic polynomials. With some algebraic manipulations, these polynomials are given in a simplified form in (3). Suppose that there exists at least one $\rho^{\star} \in[0,1]$ which is a root of one of the two polynomials $\left\{f_{1}(\rho), f_{2}(\rho)\right\}$. (In other words we are assuming that there exists at least one $\rho^{\star}$ in the interval $[0,1]$ which is either a solution to $-\rho=g(\rho)$ or a solution to $\rho=g(\rho)$.) Then if at any time $\left|\rho_{t}\right|=\rho^{\star}$ and $\rho^{\star}$ is a root of $f_{1}(\rho)$ or $f_{2}(\rho)$, then will we have $\left|\rho_{t+k}\right|$ equal to $\rho^{\star}$ for all $k \geq 0$. If $\rho^{\star}$ is a root of $f_{1}(\rho)$ (or a solution to $-\rho=g(\rho))$, then $\rho_{t+k}=(-1)^{k} \rho^{\star}$; and if $\rho^{\star}$ is a root of $f_{2}(\rho)$ (or a solution to $\rho=g(\rho)$ ), then $\rho_{t+k}=\rho^{\star}$ for all $k \geq 0$. Therefore in order to prove Lemma 6 , we need to show existence of a root in the interval $[0,1]$ of at least of one of the two polynomials $\left\{f_{1}(\rho), f_{2}(\rho)\right\}$.

We first consider the polynomial $f_{1}(\rho)$. Since $f_{1}(\rho)$ is a continuous function, it will have at least one root in the interval $[0,1]$ if it changes sign within this interval. That is at least one root exists in $[0,1]$ if we have either $\left\{f_{1}(0) \geq 0, f_{1}(1) \leq 0\right\}$ or $\left\{f_{1}(0) \leq 0, f_{1}(1) \geq 0\right\}$. Вy evaluating $f_{1}(\rho)$ for $\rho=0,1$, we get

$$
\begin{aligned}
& f_{1}(0)=1+\frac{N\left(2 h-\rho_{z}\right)}{2 h^{3} P}, \\
& f_{1}(1)=-\frac{P N\left(h^{2}\left(\rho_{z}+1\right)+2 h\left(\rho_{z}+1\right)\left(\rho_{z}+1\right)+2 N^{2}\right)}{2 h^{3} P^{2}},
\end{aligned}
$$

where $\rho_{z} \in[-1,1]$ and $P, N, h>0$. For all $\rho_{z} \in[-1,1]$, we have $f_{1}(1)<0$. Further $f_{1}(0)>0$ for $-1 \leq \rho_{z} \leq$ $2 h\left(1+\frac{h^{2} P}{N}\right)$, and $f_{1}(0)<0$ for $2 h\left(1+\frac{h^{2} P}{N}\right)<\rho_{z} \leq 1$. Therefore the existence of a root of $f_{1}(\rho)$ in the interval $[0,1]$ is guaranteed for all $\rho_{z} \in\left[-1,2 h\left(1+\frac{h^{2} P}{N}\right)\right]$. Since we could not show the existence of a root in $[0,1]$ of $f_{1}(\rho)$ when $\rho_{z}>2 h\left(1+\frac{h^{2} P}{N}\right)$, we now investigate the polynomial $f_{2}(\rho)$ for all $\rho_{z}>2 h\left(1+\frac{h^{2} P}{N}\right)$. By evaluating $f_{2}(\rho)$ for $\rho=0,1$, we get

$f_{2}(0)=-\left(1+\frac{N\left(2 h-\rho_{z}\right)}{2 h^{3} P}\right), f_{2}(1)=\frac{N(1+h)^{2}\left(\rho_{z}-1\right)}{2 h^{3} P}$.

We observe that $f_{2}(1) \leq 0$ for $\rho_{z} \in[-1,1]$, and $f_{2}(0)>0$ for $\rho_{z}>2 h\left(1+\frac{h^{2} P}{N}\right)$. Therefore a root in $[0,1]$ of $f_{2}(\rho)$ exists when $\rho_{z}>2 h\left(1+\frac{h^{2} P}{N}\right)$. Hence we have shown the existence of a root in the interval $[0,1]$ of at least of one of the two polynomials for all values of $\left\{P, h, N, \rho_{z}\right\}$. Further we observe that $f_{2}(1)=0$ for $\rho_{z}=1$, from which Remark 4 follows. 